\title{
ACTIVE SURFACE POTENTIAL CONTROL FOR ARTIFICAL ION PUMPS

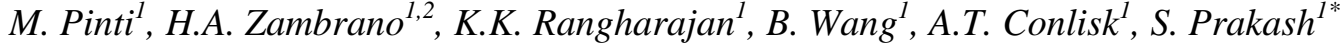 \\ ${ }^{1}$ The Ohio State University, Columbus, Ohio, USA \\ ${ }^{2}$ Universidad de Concepción, Concepción, Biobío, Chile
}

\begin{abstract}
Embedded electrodes in $16 \mathrm{~nm}$ deep, $30 \mu \mathrm{m}$ wide, and $0.5 \mathrm{~cm}$ long nanochannels were used to pump aqueous electrolyte solutions. Variations in the surface potential due to actively controlled electrodes led to flow gating and flow reversal in nanofluidic channels demonstrated through current-voltage experimental data. Active control of surface potential for pumping aqueous electrolyte solutions provides a key advance towards development of an artificial ion pump.
\end{abstract}

\section{INTRODUCTION}

Ion channels and ion-pumps form the basis of nearly all cellular communication and electrolyte transport for maintaining essential cell functions [1]. Numerous efforts have been made to develop 'smart' nanostructures to understand and implement operational principles of artificial ion channels and ion pumps for applications in bio- and chemical sensing [2], drug delivery [1], and energy conversion systems [3]. There are three main requirements for ion channels and ion pumps to be implemented: (i) sensing of species for (ii) flow gating of the ionic species (e.g., $\mathrm{K}^{+}, \mathrm{Na}^{+}$, or $\mathrm{Ca}^{2+}$ ), and (iii) selectively pumping the species of interest in a preferential direction. Significant progress has been made in requirements (i) and (ii) leading to potential development of artificial ion channels, with almost all previous reports using solid-state nanopores in polymers with chemically functionalized surfaces [2]. Some previous reports also show using flow-FETs featuring surface electrodes in nanochannels to modulate nanoflows $[4,5]$. Here, experimental current-voltage (I-V) data shows use of asymmetrically distributed surface potential through actively controlled embedded electrodes for pumping aqueous electrolytes where flow gating and flow reversal was achieved key advances towards developing an artificial ion pump.

\section{METHODOLOGY}

Nanofluidic channel networks were fabricated with aspect ratios as low as 0.0005 to enable nanoscale effects similar to biological systems; while providing high throughput and benefits of 1-D theoretical analysis. The devices were fabricated in borosilicate glass substrates using standard UV lithography and wet etching, with unique advances previously reported by our team [6]. The nanochannels were sealed using a thin $(<1 \mu \mathrm{m})$ PDMS (poly dimethylsiloxane) adhesion layer supported on a glass cover with patterned $\mathrm{Au}$ electrodes $(\sim 30 \mu \mathrm{m}$ wide, $15 \mathrm{~nm}$ thick, and distributed asymmetrically i.e. with non-uniform spacing along length of nanochannel). Two microfluidic channels with $10 \mu \mathrm{m}$ depth and either 50 or $100 \mu \mathrm{m}$ width serve as fluidic reservoirs to the bank of these ultra-low aspect ratio nanochannels with a critical dimensions of $16 \mathrm{~nm}$ (Fig. 1). Electrolyte solutions with $\mathrm{KCl}$, $\mathrm{NaCl}$, or $\mathrm{CaCl}_{2}$ in DI water at concentrations varied between $0.1 \mathrm{mM}$ to $100 \mathrm{mM}$ were used as working fluids.

\section{RESULTS}

With no surface electrode potential, nanochannel conductance, given by the ratio of current to applied axial potential $(\mathrm{I} / \mathrm{V})$, remains constant at low electrolyte concentrations as measured by comparable currents for $0.1 \mathrm{mM}$ and $1 \mathrm{mM} \mathrm{KCl}$ (Fig. 2A). However, as the concentration increases to $10 \mathrm{mM}$,

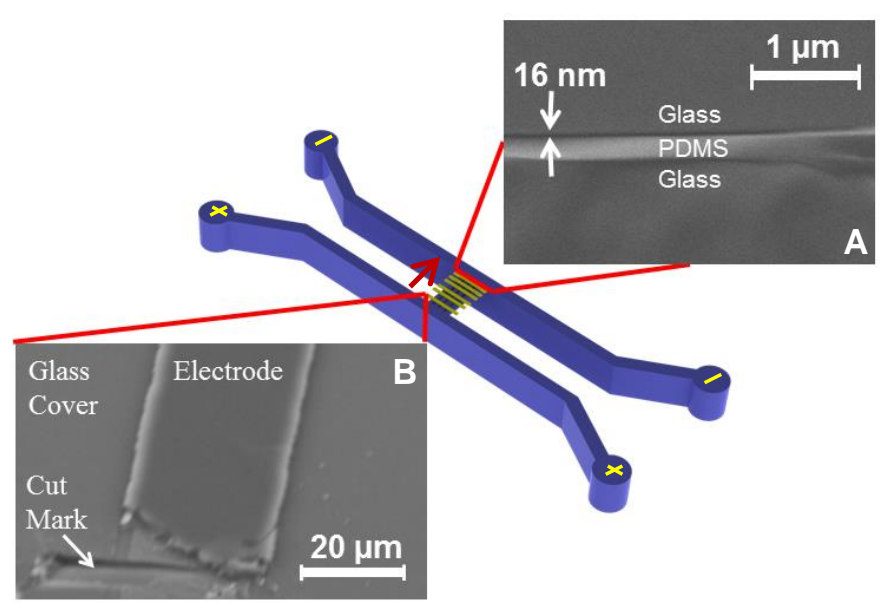

Figure 1: Schematic for the geometric layout of the active ion pumping device, in a hybrid micro/nanofluidic configuration. Two microchannels connect a bank (3-6) of $16 \mathrm{~nm}$ deep x $30 \mu \mathrm{m}$ wide $x 0.5 \mathrm{~cm}$ long nanofluidic channels. An array of non-uniformly spaced Au electrodes are patterned on a glass cover and isolated from the liquid by PDMS, which is then used as an adhesion layer to bond and seal the nanochannels. The "+" and "-" signs indicate the anode and cathode used to apply the axial potential respectively. The red arrow indicates the direction of net flow. [A] SEM image showing a partial cross section of a $16 \mathrm{~nm}$ deep channel to confirm that channels are bonded and open for flow. The 3-layers show the glass substrate, PDMS adhesion layer, and glass cover. Note, the aspect ratio of the nanochannel makes it impossible to view the entire cross-section in one frame. [B] SEM image of a gold electrode on a glass cover before it is embedded in PDMS and sealed to form the top of the channel. A thicker electrode (50 $\mathrm{nm}$ compared to $15 \mathrm{~nm}$ in a typical bonded device) is shown here to allow the full width and finite thickness to be observed in one frame. The cover with the electrode was cut for imaging purposes leading to a partial ripping of the Au film.

increase in current implies conductance values tend towards those expected from the bulk conductivity, matching previous reports for $\mathrm{KCl}$ in nanofluidic channels $[4,5,7]$. Figure $2 \mathrm{~B}$ shows that iontype can have a significant impact on the I-V data, with the measured current scaling as a function of valence. Current data from $10 \mathrm{mM} \mathrm{KCl}$ and $10 \mathrm{mM} \mathrm{NaCl}$ shows no significant difference despite approximately $40 \%$ difference in ionic mobility of $\mathrm{K}^{+}$and $\mathrm{Na}^{+}$(values of 7.63 and $5.18 \times 10^{-8} \mathrm{~m}^{2} \mathrm{~V}^{-1} \mathrm{~s}^{-1}$, respectively [8]). Comparison of the slopes of the linear portion of the I-V data in Figure 2B indicates $\mathrm{NaCl}$ and $\mathrm{KCl}$ have nearly 5x higher conductance compared to $\mathrm{CaCl}_{2}$ where the ionic mobility of $\mathrm{Ca}^{2+}$ is higher than $\mathrm{Na}^{+}$(with a value of $6.15 \times 10^{-8} \mathrm{~m}^{2} \mathrm{~V}^{-1} \mathrm{~s}^{-1}$ for $\mathrm{Ca}^{2+}$ ).

The total current through a nanofluidic channels is the summation of contributions due to diffusion, conduction, and convection. Neglecting diffusion, the total current through the nanofluidic channel is given by

$$
I=\int_{0}^{h} F w \sum_{i} z_{i}\left(-\Omega_{i} c_{i} \nabla \phi+c_{i} \vec{u}\right) d x
$$


where $F$ is Farday's constant, $c_{i}$ is the concentration of the species in the nanochannel, $z_{i}$ is the valence, and $\Omega_{i}$ is the ionic mobility given by $\Omega_{i}=z_{i} F D_{i} / R T$ [8]. The potential gradient, $\nabla \varphi$, is given by $-V_{\text {axial }} / L_{\text {channel }}$ in long channels with the bias configuration shown in Fig. 1 [9]. Considering the values for the valence and ionic mobility, the current values shown in Fig. $2 \mathrm{~B}$ can be explained by variations in $c_{i}$ and $\overrightarrow{\boldsymbol{u}}$, indicating the difference in transport observed for different cations is dominated by electrostatic interactions between the electrolyte and the charged surface.

Microfluidic particle image velicometry measurements show that the presence of trace amounts of calcium reduces the
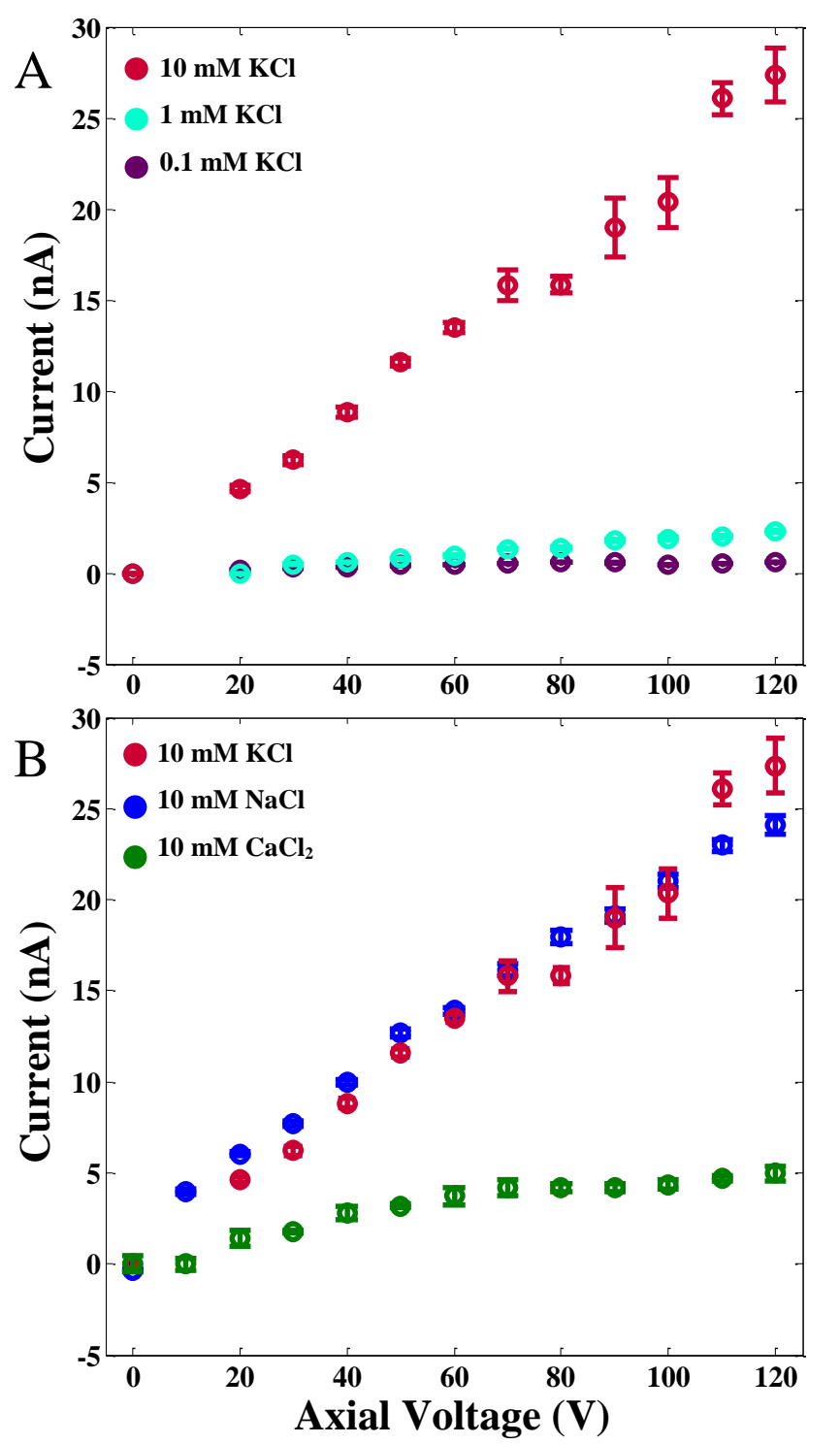

Figure 2: [A] I-V data for $\mathrm{KCl}$ confirming operation of a $16 \mathrm{~nm}$ deep nanochannel device. Comparable current and, therefore, conductance is observed for $0.1 \mathrm{mM}$ and $1 \mathrm{mM}$ while the $10 \mathrm{mM}$ case shows increased current and, therefore, conductance expected in these types of devices in agreement with previous reports. [B] I-V data shows influence of distinct cations on total measured current in absence of a gate potential. At 10mM, KCl and $\mathrm{NaCl}$ show comparable values for current. Conductance (slope of I-V curve) of $\mathrm{CaCl}_{2}$ filled channels is nearly $5 \times$ lower than $\mathrm{NaCl}$ and $\mathrm{KCl}$. magnitude of the electroosmotic velocity in $\mathrm{KCl} / \mathrm{CaCl}_{2}$ mixtures compared to pure $\mathrm{KCl}$. The effect is attributed to reduction of the surface potential due to calcium adsorption to the wall [10], with similar results reported for $\mathrm{KCl} / \mathrm{CaCl}_{2}$ mixtures in nanofluidic pores [11]. To our knowledge, this is the first direct comparison of $\mathrm{CaCl}_{2}$ to $\mathrm{NaCl}$ and $\mathrm{KCl} \mathrm{I-V}$ data and further investigation is required to fully explain the observed trends.

By controlling the applied electrode potential for a fixed streamwise or axial potential, reversal of net measured current in the nanochannel was observed, demonstrating a reversal in direction of net ionic flow (Fig. 3). In fact, gating to stop flow as indicated by nearly zero measured current, and then reversing polarity of measured current is seen in Fig. 3 for $1 \mathrm{mM} \mathrm{NaCl}$ electrolyte solution as a representative case. As the measured current is a total current with contributions from net diffusion, conduction, and convection, the reversal in measured current polarity indicates the reversal of the direction of net ion transport. Leakage current through the PDMS insulation layer was negligible, indicating extraneous current paths do not affect the data shown in Fig. 3.

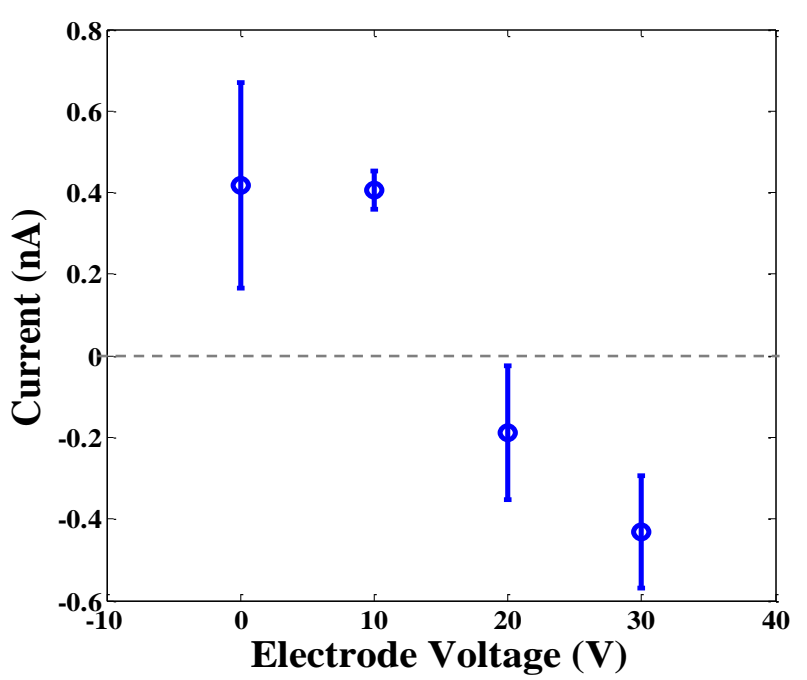

Figure 3: At a fixed axial potential of $15 \mathrm{~V}$ using $1 \mathrm{mM} \mathrm{NaCl}$ electrolyte, the electrode or gate potential was increased leading to a decrease in current (and therefore conductance) until the channel is effectively "closed" and allows no net flow through the channel. Further increasing the voltage leads to a change in the polarity of the current indicating that the net ion transport within the nanochannel has reversed direction.

Fixing the surface electrode potential to $-10 \mathrm{~V}$, pumping of $1 \mathrm{mM} \mathrm{CaCl}{ }_{2}$ was observed with no axial applied potential as indicated by a positive $67 \mathrm{pA}$ current (Fig. 4). Figure 4 shows a representative case where the surface electrode alone was capable of pumping an aqueous electrolyte solution in the nanofluidic channel. An axial potential of $130 \mathrm{~V}$, more than an order of magnitude higher than the surface electrode potential used here, is required to drive a $67 \mathrm{pA}$ current for $1 \mathrm{mM} \mathrm{CaCl}_{2}$ without surface electrodes.

Flow gating and current reversal can also be observed by varying the axial potential. The current reduces in magnitude as the axial potential becomes increasingly negative where full flow gating is observed at $-70 \mathrm{~V}$ and flow reversal is observed at larger $(>-70 \mathrm{~V})$ negative axial potentials. Further investigation is required to determine the origin of the non-linearities observed in the IV data in Figure 4. 


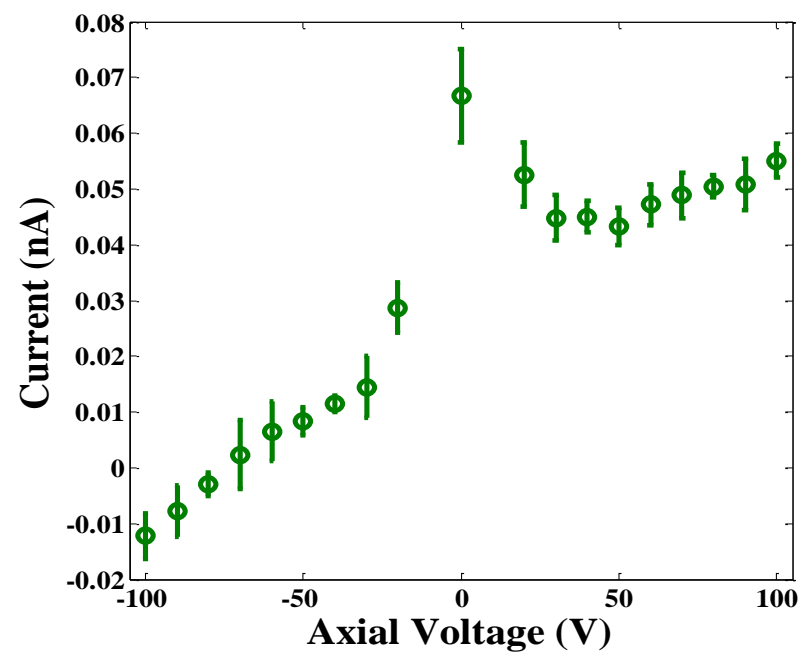

Figure 4: Pumping of $1 \mathrm{mM} \mathrm{CaCl} \mathrm{Cl}_{2}$ electrolyte was observed with a potential of $-10 \mathrm{~V}$ applied to the surface electrode and no axial potential as indicated by a positive 67 pA current. An axial potential of $130 \mathrm{~V}$, more than an order of magnitude higher than the surface electrode potential used here, is required to drive a $67 \mathrm{pA}$ current for $1 \mathrm{mM} \mathrm{CaCl}$ without surface electrodes. Applying a net negative axial potential led to a decrease in current until the channel is effectively "closed" and allows no net flow through the channel at $\sim 70 \mathrm{~V}$. Further increasing the magnitude of the negative voltage led to a reversal in net ionic transport as indicated by a negative measured current.

\section{CONCLUSIONS}

We have demonstrated for the first time reversal of net ionic transport in a nanofluidic channel through application of a transverse electric field via an embedded surface electrode. The configuration presented here allows for tunable control of the direction of transport in the nanofluidic channel, where gating and flow reversal have been demonstrated. The surface electrode alone pumps the electrolyte solution using an applied potential that is one order of magnitude lower than a streamwise or axial potential to achieve similar electrokinetic pumping, as observed due to measured currents.

\section{ACKNOWLEDGEMENTS}

The authors acknowledge the staff at Nanotech West Laboratories for assistance with equipment during fabrication and characterization of devices. We also acknowledge partial financial support from the US Army Research Office (ARO), and the National Science Foundation (NSF). The authors wish to acknowledge computational support from the Ohio Supercomputer Center (OSC).

\section{REFERENCES}

[1] R. Duan, F. Xia and L. Jiang, "Constructing Tunable Nanopores and Their Application in Drug Delivery", ACS Nano, 7, 10 (2013) pp. 8344-8349.

[2] X. Huo, W. Guo and L. Jiang, "Biomimetric smart nanopores and nanochannels", Chemical Society Reviews, 40, (2011) pp. 2385-2401.

[3] L. Wen, X. Hou, Y. Tian, F.-Q. Nie, Y. Song, J. Zhai and L. Jiang, "Bioinspired Smart Gating of Nanochannels Toward Photoelectric-Conversion Systems", Advanced Materials, 22, 9 (2010) pp. 1021-1024.

[4] R. Karnik, R. Fan, M. Yue, D. Li, P. Yang and A. Majumdar, "Electrostatic Control of Ions and Molecules in Nanofluidic Transistors", Nano letters, 5, 5 (2005) pp. 943-948.

[5] R. Fan, S. Huh, R. Yan, J. Arnold and P. Yang, "Gated proton transport in aligned mesoporous silica films", Nature Materials, 7, (2008) pp. 303-307.

[6] M. Pinti, T. Kambham, B. Wang and S. Prakash, "Fabrication of Centimeter Long, Ultra-Low Aspect Ratio Nanochannel Networks in Borosilicate Glass Substrates", Journal of Nanotechnology in Engineering and Medicine, 4, 2 (2013) pp. $1-7$.

[7] D. Stein, M. Kruithof and C. Dekker, "Surface Charge Governed Ion Transport in Nanofluidic Channels", Physical Review Letters, 93, 3 (2004) pp. 035901 1-4.

[8] A. T. Conlisk, Essentials of Micro- and Nanofluidics: Canbridge University Press, 2013.

[9] I. Vlassiouk, S. Smirnov and Z. Siwy, "Ionic Selectivity of Single Nanochannels", Nano letters, 8, 7 (2008) pp. 1978-1985.

[10] S. Datta, A. T. Conlisk, H. F. Li and M. Yoda, "Effect of divalent ions on electroosmotic flow in microchannels", Mechanics Research Communication, 36, (2009) pp. 65-74.

[11] Z. S. Siwy, M. R. Powell, A. Petrov, E. Kalman, C. Trautmann and R. S. Eisenberg, "Calcium-Induced Votlage Gating in Single Conical Nanopores", Nano letters, 6, 8 (2006) pp. 17291734.

\section{CONTACT}

*S. Prakash, tel: +1-614-688-4045; prakash.31@osu.edu 